ORIGINAL ARTICLE

\title{
Estimated date of delivery in Chihuahua breed bitches, based on embryo-fetal biometry, assessed by ultrasonography
}

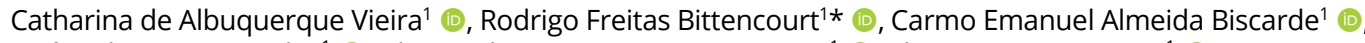

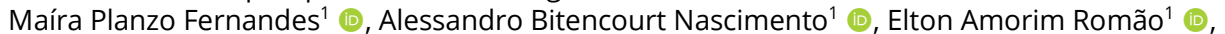

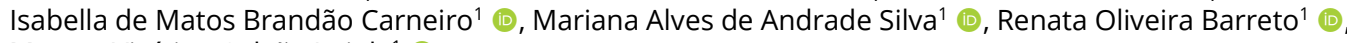
Marcus Vinícius Galvão Loiola' [1]
\end{abstract}

'Setor de Reprodução Animal e Obstetrícia, Hospital Veterinário, Escola de Medicina Veterinária e Zootecnia, Universidade Federal da Bahia, Salvador, BA, Brasil

How to cite: Vieira CA, Bittencourt RF, Biscarde CEA, Fernandes MP, Nascimento AB, Romão EA, Carneiro IMB, Silva MAA, Barreto RO, Loiola MVG. Estimated date of delivery in Chihuahua breed bitches, based on embryofetal biometry, assessed by ultrasonography. Anim Reprod. 2020;17(3):e20200037.

https://doi.org/10.1590/1984-3143-AR2020-0037

\begin{abstract}
Due to the peculiarities of the reproductive cycle of the female dog, which makes it difficult to accurately ascertain the date of conception, it may be challenging to precisely estimate the gestational age in bitches. Using fetal measurements obtained by ultrasound, it is possible to estimate the gestational age in dogs; however, due to the differences in size of the various breeds, such estimates may have a significant standard deviation, which represents less accuracy when specifying the date of birth. The purpose of this study was to evaluate pregnant female Chihuahuas, establishing relations between the fetal dimensions measured by ultrasound and the remaining time until delivery. Using 13 pregnant female Chihuahuas, weekly ultrasound assessments and measurements were performed, of the inner chorionic cavity, cranial-caudal length, biparietal diameter, abdominal diameter and thoracic diameter. Such parameters were retroactively correlated to the date of delivery, and linear regressions were established between the gestational measurements and remaining days until delivery. The fetal measurement presenting the highest correlation $(r=0.99 ; \mathrm{P}<0.0001)$ and reliability $\left(\mathrm{R}^{2}=0.98, P<0.0001\right)$ with the probable date of delivery was the biparietal diameter, a measurement that can be easily and safely obtained and, when applying a specific formula $(Y=-15.46 X 1+38.72)$, can accurately predict the date of delivery in Chihuahua female dogs.
\end{abstract}

Keywords: canine, gestational age, pregnancy, ultrasound.

\section{Introduction}

In the canine species, the time elapsed from the date of mating does not necessarily coincide with the gestational time. This is due to the long period of sexual receptivity of the female bitch, such that the bitch may stand to be bred either long before of well after ovulation occurs. Additionally, variation in length between mating and delivery is dependent on the prolonged viability of the sperm in the reproductive tract, which leads to a variation in the estimate of the gestational duration from 57-68 days after copulation (Concannon and Lein, 1989).

In fact, parturition occurs $65 \pm 1$ days after LH peak and $63 \pm 1$ days after ovulation (Concannon et al., 1983). However, detection of LH peak and the ultrasonographic follow-up of ovulation are unpractical because of the requirement of daily examinations (Hase et al., 2000).

*Corresponding author: rfb@ufba.br

Received: April 10, 2020. Accepted: July 6, 2020.

Financial support: None.

Conflicts of interest: The authors have no conflict of interest to declare.

(c) (i) Copyright (c) The Author(s). This is an Open Access article distributed under the terms of the Creative Commons Attribution License, which permits unrestricted use, distribution, and reproduction in any medium, provided the original work is properly cited. 
Therefore, in order to plan and monitor the deliveries, a more practical method for establishing the gestational age is required.

B-mode ultrasonography is a semiological means of relatively low cost, is non-invasive, harmless to the bitch and puppies, and provides valuable information about the fetal development (Papp and Fekete, 2003). With ultrasonography, it is possible to estimate the gestational age, by means of formulas that relate fetal measurements to the gestational period, in addition to assessing the fetal viability (Beccaglia et al., 2016).

Ultrasonographic assessment of the gestational age in female dogs is somewhat limited by the great variability in size and body conformation of different breeds of dogs. Therefore, there have been attempts to establish more specific formulas for different breeds, sizes and cranial conformations, to better assist clinicians in monitoring the gestational age of female dogs (Camargo et al., 2011).

Several authors have studied the relationship between fetal biometrics and the development of the pregnancy in different breeds of different sizes, such as the Boxer (Silva et al., 2007), Yorkshire Terrier (Jabin et al., 2007), American Cocker Spaniel and Chow-Chow (Melo et al., 2006), Rottweiler (Teixeira et al., 2009), German Shepherd (Groppetti et al., 2015; Cecchetto et al., 2017; Socha and Janowski, 2017), observing differences between the biometric formulas used for each size or breed of the studied dogs.

In the authors clinical experience, was observed that the formula used to estimate the gestational age $(G A=$ biparietal diameter $\times 15+20)$, described by Nyland and Mattoon (2002), for all breeds of dogs, did not correspond to the correct gestational age at the end of the pregnancy of female Chihuahua dogs.

The Chihuahua breed is widely distributed globally and is considered the smallest dog breed in the world $(\mathrm{FCl}, 2010)$, with a relatively large skull (Traas, 2008) and a high risk of dystocia (Gaudet, 1985; Bergström et al., 2006; O'Neill et al., 2017). Due to these specific biometric traits, it is difficult to apply general formulas to precisely determine the pregnancy duration, which is necessary to determine due date and make plans for possible interventions in cases of dystocia.

To our knowledge, there are no currently available published studies that use fetal biometry to estimate the gestational age specific for dogs of the Chihuahua breed. Thus, the purpose of this study was to monitor pregnant Chihuahuas and to establish formulas that will relate fetal measurements to the gestational age, and determine the expected date of delivery.

\section{Methods}

The experiment was conducted in the Animal Reproduction and Veterinary Obstetrics Department of the Hospital of Veterinary Medicine, School of Veterinary Medicine and Animal Science of the Federal University. The study was approved by the local Ethics Committee on Animal Use (CEUA), under protocol EMEVZ $n^{\circ}$. 10/2017.

Thirteen pregnant Chihuahuas were used (eight long-haired and five short-haired), five primiparous and eight multiparous, between 1-3 years old and a body weight between 2-4 kg. The females belonged to both commercial kennels and hobby breeders, and were mated with males of the same breed using natural mating or artificial insemination techniques, according to the reproductive program of each breeder.

To perform the obstetric ultrasound exam, the dogs were positioned and restrained in dorsal decubitus on a padded surface specific for the purpose, without sedation, and the females were prepared with abdominal trichotomy from the xiphoid cartilage to the pubis, extending laterally to the renal region.

A portable ultrasound device (Z5, Mindray Z5) was used, equipped with a linear and a micro-convex transducer, with frequencies between 6.5-8.5 $\mathrm{MHz}$. The type of transducer, as well as the frequency used, were chosen at the time of examination, depending on the gestational phase and structure to be assessed. To improve the transmission of ultrasound waves, ultrasound gel was used on the skin of the region to be examined. 
The females went through weekly ultrasound evaluations, starting between 15-30 days after the date of the latest mating, until the day of delivery, totaling between 3-6 exams per female. All analyses were performed by the same experienced ultrasonographer and images with the measurements were later evaluated by a second examiner.

The following fetal/embryonic and extra-fetal structures were assessed: biparietal diameter (BPD): obtained from the external measure of the greater distance between the parietal bones, in the transverse view of the skull (Son et al., 2001; Groppetti et al., 2015). Abdominal Diameter (ABD): measured using the section of the abdomen at the largest diameter at stomach's height (Son et al., 2001). Thoracic diameter (TD): measurement performed on the greatest distance from the cross section of the chest, at the point of the last rib (Jabin et al., 2007). Craniocaudal length $(C C L)$ : obtained by the measurement of length from the most cranial point on the skull to the caudal edge to the perineum (Son et al., 2001; Beccaglia and Luvoni, 2006). Inner diameter of the chorionic cavity (IDCC): corresponding to the perpendicular distances between the inner walls of the chorionic cavity, in its largest and smallest diameters, determined by taking the mean of those two ICC diameters made at $90^{\circ}$ angles from one side of the trophoblastic decidual reaction to the other (Beccaglia and Luvoni, 2006; Groppetti et al., 2015) (Figure 1).
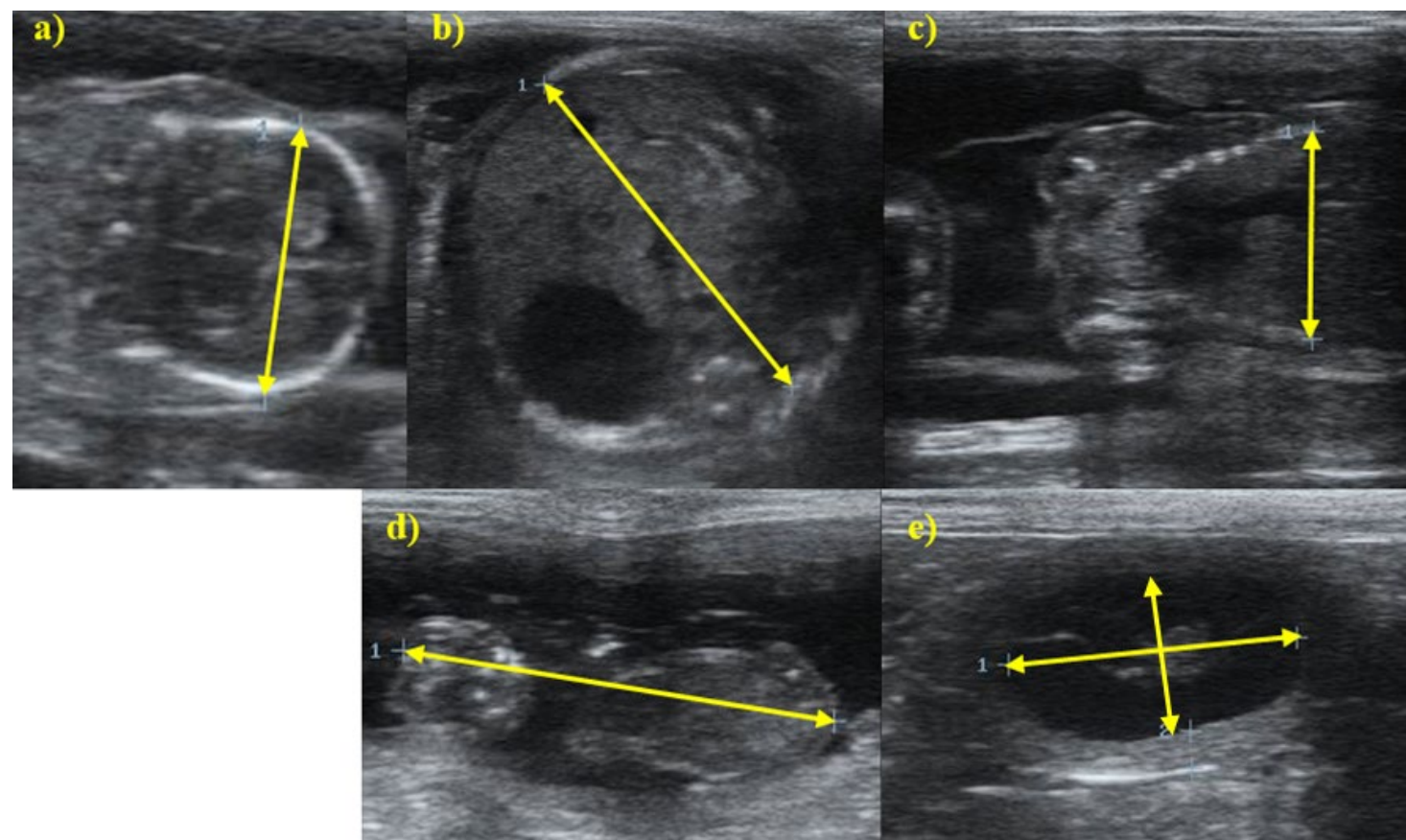

Figure 1. Ultrasound images of the measurements taken in fetal and non-fetal structures in pregnant Chihuahua female dogs. (a) ultrasound measurement of the biparietal diameter - canine fetus of the Chihuahua breed 16 days before delivery; (b) ultrasound measurement of abdominal diameter - canine fetus of the Chihuahua breed six days before delivery; (c) ultrasonographic measurement of the thoracic diameter - canine fetus of the Chihuahua breed 16 days before delivery; (d) ultrasonographic measurement of the craniocaudal length - canine embryo of the Chihuahua breed 32 days before delivery; (e) ultrasonographic measurement of the diameter of the internal chorionic cavity - fetal gallbladder of the female Chihuahua 35 days before delivery. B-mode ultrasonography, equipment Mindray Z5, linear transducer, $7.5 \mathrm{MHz}$.

The data obtained from the measurements (BPD, ABD, TD, CCL and IDCC) were presented as averages of all fetuses examined during the evaluation of each bitch and were retroactively related to the remaining days before parturition (DBP), from the date that labor occurred as reported by the breeder, considered day zero. We performed a simple linear regression and an ANOVA analysis, using the program IBM SPSS Statistics (IBM Corp. Released 2017. IBM SPSS Statistics for Windows, Version 25.0. IBM Corp), using the equation $y=a x+b$, where " $y$ " corresponds to the days before delivery, " $x$ " is the measurement of the structure, " $a$ " is the constant coefficient and "b" the first order coefficient. 


\section{Results}

Of the thirteen monitored pregnancies, 43 living puppies were born, all from natural and a term delivery, no cases of fetal mummification or stillborns. The size of the litters ranged from 1-7 puppies, with an average of 3.2 offspring per female. In total, 64 ultrasound examinations were performed, and on each assessment, it was possible to individually evaluate until five fetuses.

It was possible to collect the measurements between the 44rd day prior to delivery and the day of delivery, and all evaluated parameters showed a significant correlation $(P<0.05)$ to the gestational age, demonstrating that the fetal parameters of the BPD, TD, ICCD and ABD have higher correlation with the days until delivery than the craniocaudal length, as detailed in Table 1.

Table 1. Gestational parameters $(X)$, the minimum and maximum values of the averages of each measurement, the interval of days before parturition (DBP), linear regression equation of days until delivery $\left(\eta\right.$, correlation coefficient $(r)$, coefficient of determination $\left(R^{2}\right)$ and estimated standard deviation (SD) corresponding to the structures assessed by B mode ultrasonography in thirteen pregnant Chihuahua females.

\begin{tabular}{cccccccc}
$\begin{array}{c}\text { Gestational } \\
\text { Parameter } \\
(\boldsymbol{X})\end{array}$ & $\begin{array}{c}\text { Minimum and } \\
\text { maximum } \\
\text { values }(\mathbf{c m})\end{array}$ & DBP & $\boldsymbol{Y}$ & $\boldsymbol{r}$ & $\boldsymbol{R}^{2}$ & SD & p-value \\
\hline $\mathrm{IDCC}(X 1)$ & $0.43-3.08$ & $44-23$ & $-7.51 \times 1+46.09$ & 0.96 & 0.93 & 1.76 & $<0.0001$ \\
\hline $\mathrm{CCL}(X 2)$ & $0.83-4.50$ & $34-20$ & $-2.63 \times 2+34.51$ & 0.87 & 0.75 & 2.00 & $<0.0001$ \\
\hline $\mathrm{BPD}(X 3)$ & $0.91-2.48$ & $25-0$ & $-15.46 \times 3+38.72$ & 0.99 & 0.98 & 1.21 & $<0.0001$ \\
\hline $\mathrm{TD}(X 4)$ & $0.86-3.43$ & $25-0$ & $-10.96 \times 4+33.50$ & 0.96 & 0.92 & 2.18 & $<0.0001$ \\
\hline $\mathrm{ABD}(X 5)$ & $1.10-4.10$ & $25-0$ & $-8.61 \times 5+35.28$ & 0.95 & 0.89 & 2.55 & $<0.0001$ \\
\hline
\end{tabular}

Fetal/embryonic and extra-fetal structures assessed by ultrasound: inner diameter of the chorionic cavity (IDCC); craniocaudal length (CCL); biparietal diameter (BPD); thoracic diameter (TD) and abdominal diameter (ABD).

It can be seen that the equations generated by the linear regression of the different measures studied were statistically validated $(\mathrm{P}<0.0001)$. In addition, it was observed that all equations had high coefficient of determination $\left(R^{2}\right)$, which represents the high degrees of reliability of the formulas. The biparietal diameter measure (BPD) showed the highest significant correlation $(r=0.99, P<0.0001)$ with the days until delivery (DBP). For this measure, it was also observed that the generated formula $(y=-15.46 \times 3+38.72)$ presented the highest determination factor with $\mathrm{R}^{2}=0.98(\mathrm{P}<0.0001)$, which means that through it one can explain $98 \%$ of the DBP events in the Chihuahua bitches. The other $2 \%$ is the statistical error, which is probably included in the standard deviation (SD) generated by the formula, of \pm 1.21 days.

The variables showed a linear distribution, increasing with the development of the pregnancy and consequent decrease in the number of days until delivery. Scatter charts were drawn up with their respective functions of linear regression by correlating the variable with the DBP (Figures 2-6). 


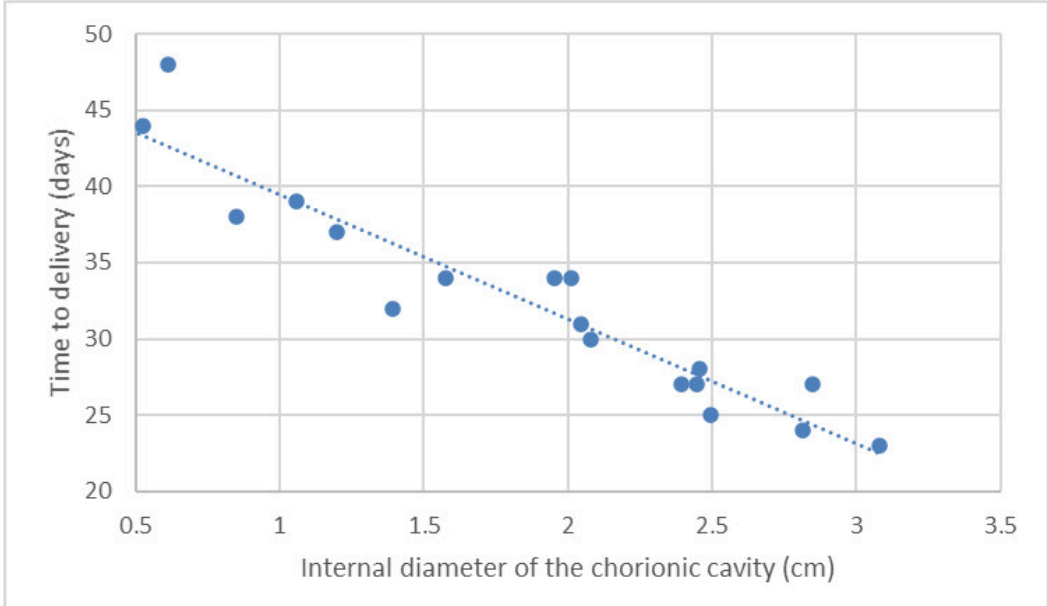

Figure 2. Scatter chart and regression line of the function of days before parturition (DBP; $\gamma$ ) in relation to the ultrasonographic measurement of the diameter of the internal chorionic cavity (ICCD; X1) on canine fetuses of the Chihuahua breed.

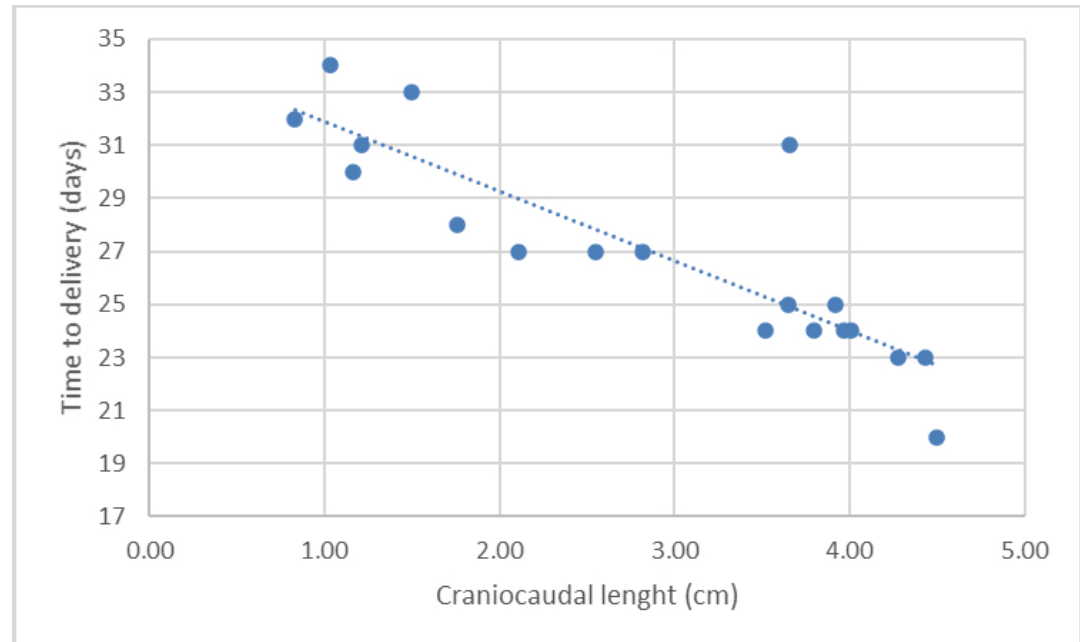

Figure 3. Scatter chart and regression line of the function of days before parturition (DBP; $\bigvee$ ) in relation to the ultrasonographic measurement of the craniocaudal length $(C C L ; X 2)$ on canine fetuses of the Chihuahua breed.

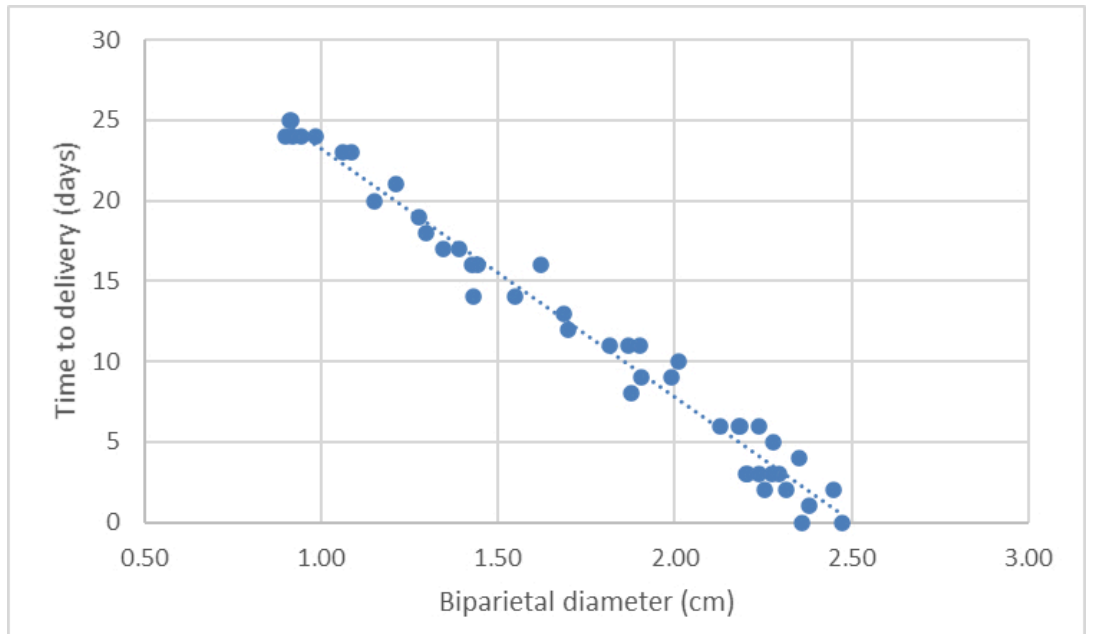

Figure 4. Scatter chart and regression line of the function of days before parturition (DBP; $\eta$ ) in relation to the ultrasonographic measurement of the biparietal diameter (BPD; X3) on canine fetuses of the Chihuahua breed. 


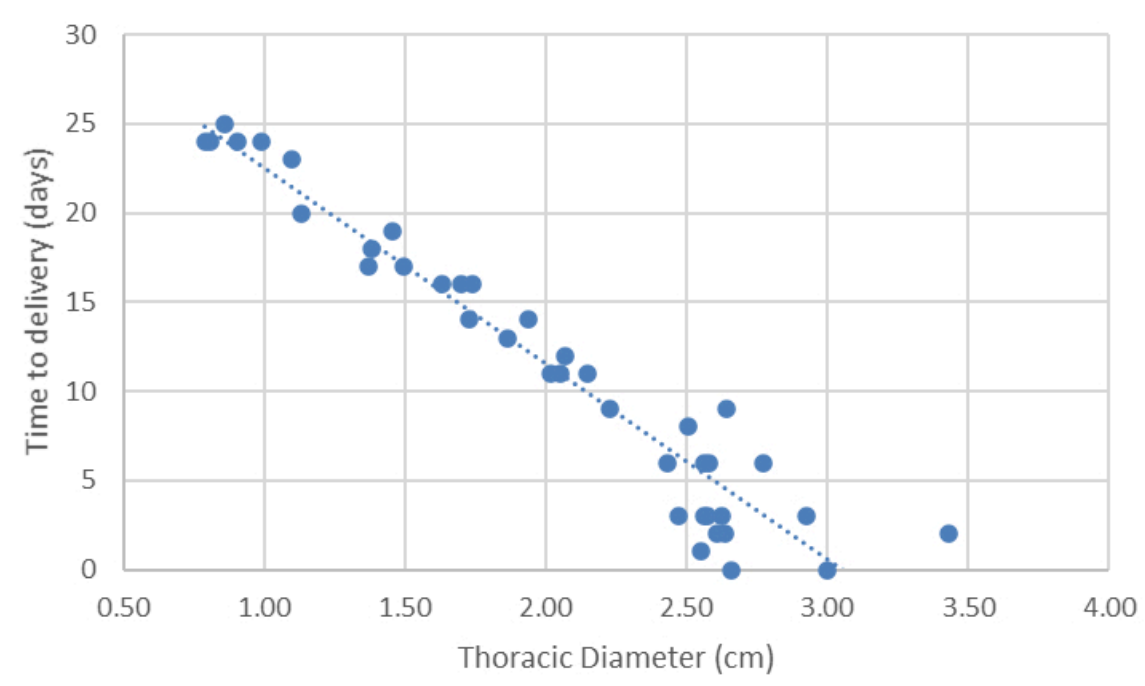

Figure 5. Scatter chart and regression line of the function of days before parturition (DBP; $\eta$ ) in relation to the ultrasonographic measurement of the thoracic diameter (TD; X4) on canine fetuses of the Chihuahua breed.

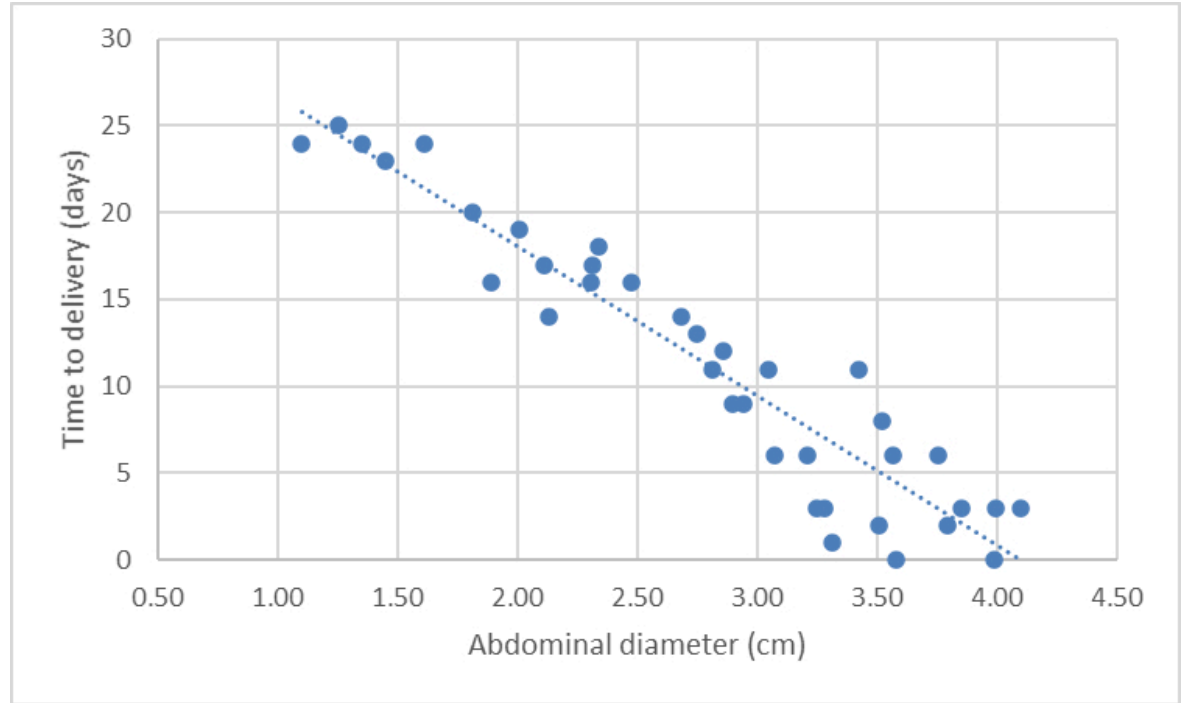

Figure 6. Scatter chart and regression line of the function of days before parturition (DBP; $\gamma$ ) in relation to the ultrasonographic measurement of the abdominal diameter (DAB; X5) on canine fetuses of the Chihuahua breed.

\section{Discussion}

Due to the difficulties mentioned earlier in estimating the exact date of conception in female dogs, without hormonal dosages or vaginal cytology, to estimate the gestational age based only on the date of mating is quite inconsistent (Luvoni and Grioni, 2000). Therefore, the study was designed to estimate the number of days until delivery. Several studies have found highlysignificant correlations between the fetal measurements and the number of days until delivery in specific breeds of dogs of varying sizes, however, none of these studies have solely studied the Chihuahua breed (Melo et al., 2006; Silva et al., 2007; Jabin et al., 2007; Teixeira et al., 2009; Camargo et al., 2011; Groppetti et al., 2015; Cecchetto et al., 2017; Socha and Janowski, 2017).

This study found a strong positive correlation between the biparietal $(r=0.99, P<0.05)$, thoracic $(r=0.96, P<0.05)$, inner chorionic cavity $(r=0.96, \mathrm{P}<0.05)$ and abdominal $(r=0.95$, 
$P<0.05)$ diameters with the remaining DBP in female Chihuahuas. In several studies, BPD was identified as the measurement with a stronger correlation with the gestational period, from the second half of pregnancy in dogs of different breeds. It was considered a reliable measurement and easy to obtain (Luvoni and Grioni, 2000; Melo et al., 2006; Jabin et al., 2007; Silva et al., 2007; Cecchetto et al., 2017).

Socha and Janowski $(2018)$ found a correlation of $0.94(P<0.05)$ of the BPD with the DBP in miniature dog breeds. The author suggests that formulas used for small and medium-sized breeds, such as those described by Luvoni and Grioni (2000) might be used in female dogs of miniature breeds. The regression formula $(y=1.6190 \times$ BPD -39.70$)$ presented in the study (Socha and Janowski, 2018) differs from the formula presented in this research and features a large variation of the values found for the Chihuahua, which highlights the need to study this breed individually and to standardize an adequate calculation.

In the investigation by Son et al. (2001), when studying the correlation between gestational age and date of delivery with the gestational parameters of two miniature breeds (Yorkshire Terrier and Maltese), a strong correlation was found ( $r=0.99)$ between the measurements of the inner chorionic cavity diameter (ICCD) and the gestational age in the period between 20-37 days of pregnancy. Similarly to Jabin et al. (2007), who calculated the volume of the chorionic vesicle, based on the ICCD, and also identified a high correlation with the gestational age $(r=0.96)$ in female Yorkshire Terriers. In the present study, it was observed a strong correlation $(r=0.96, P<0.05)$ between the ICCD and the DBP in the period between 44-27 days before delivery, presenting the ICCD as the best parameter to predict the delivery in the early half of pregnancy.

Also, in the study by Jabin et al. (2007), the CCL showed a correlation coefficient of 0.97 with the gestational age, when measured between 21-27 days until 42 days of pregnancy, which is different from this study, where a weaker correlation (0.87) was found when measuring that fetal parameter between 34-20 days before delivery. This small window of fourteen days of pregnancy to perform the measurement provided little data and, for some females, only one measurement was taken, which limits the clinical application. This is because, when fetuses grow, it bends, altering the values of this measurement. In addition, when pregnancy progresses, the transducer used in the experiment was not capable of forming an image of the entire fetal length, preventing the measurement in more advanced pregnancies.

In this study the coefficients of determination found for the equations generated with the measurements made at the end of pregnancy (BPD, TD and ABD) were always high (0.98, 0.92 and 0.89, respectively), which represents an accurate prediction of delivery. However, in advanced pregnancies, for greater accuracy in predicting the delivery onset and diagnosis of fetal distress, it is important to correlate the values found in biometric formulas with other ultrasound analyses such as organogenesis (Pieri et al., 2015) and measurement of heart rate fetal (Gil et al., 2014), or more complex studies, through Doppler ultrasound, to assess the resistivity of the umbilical artery (Giannico et al., 2015). Such assessments, associated with clinical signs, like rectal temperature, provide greater security for the professional to monitor the delivery and, especially, schedule a cesarean section.

All the female dogs experienced natural deliveries; only two dogs were assisted by a veterinary medical team, and there was no incidence of dystocia in the studied population. In some previous studies, high rates of labor dystocia were observed in the Chihuahua breed (Gaudet, 1985; Bergström et al., 2006; O’Neill et al., 2017), considered a dog of large cranial dimensions, that might need an episiotomy due to the head of the puppy being entrapped in the vulvar opening (Traas, 2008).

In the study by O'Neill et al. (2017), while assessing more than 18 thousand female dogs, the Chihuahua breed presented the highest number of cases of dystocia and was the fourth highest in terms of prevalence and risk factors for dystocia, immediately after three brachycephalic breeds (French Bulldog, Boston Terrier, and Pug). As the records presented in the literature are from other countries, it is possible that the genetic selection of the Brazilian commercial kennels made it possible to reduce this incidence, as no events of cesarean section or episiotomy have been recorded in Chihuahuas in recent years in the clinical routine of the 
hospital where the study was developed, besides the fact that this is not such a common breed in Brazil as it is in other countries.

The predisposition of the Chihuahua breed to have a dystocia justifies the need to monitor the gestational age of these females and to estimate the probable date of delivery, to prepare both the guardian and the veterinary team for the possibility of a cesarean section or assisted delivery. By knowing the date of delivery, it is possible to be attentive to any abnormal behavior, to take measures to anticipate problems that may cause fetal death or put the life of the dam at risk, or to decide to perform an elective cesarean section (Socha and Janowski, 2018).

\section{Conclusion}

The Chihuahua breed has peculiarities in the fetal biometry, making it necessary to standardize fetal measurement calculations by ultrasonography, for the better pregnancy and parturition management. The high correlations observed between the embryo-fetal measures and the remaining time until delivery, added to high factors of determination obtained by specific formulas established, especially with the BPD use, allow an accurate prediction of the date of birth in this breed.

\section{References}

Beccaglia M, Alonge S, Trovo C, Luvoni GC. Determination of gestational time and prediction of parturition in dogs and cats: an update. Reprod Domest Anim. 2016;51(Suppl 1):12-7. http://dx.doi.org/10.1111/rda.12782. PMid:27670935.

Beccaglia M, Luvoni GC. Comparison of the accuracy of two ultrasonographic measurements in predicting the parturition date in the bitch. J Small Anim Pract. 2006;47(11):670-3. http://dx.doi.org/10.1111/j.1748-5827.2006.00108.x. PMid:17076791.

Bergström A, Nødtvedt ANE, Lagerstedt AS, Egenvall A. Incidence and breed predilection for dystocia and risk factors for cesarean section in a Swedish population of insured dogs. Vet Surg. 2006;35(8):78691. http://dx.doi.org/10.1111/j.1532-950X.2006.00223.x. PMid:17187641.

Camargo NI, Berlim AT, Magalhães FJR, Manso Filho HC, Wischral A. Fetal biometry in Shih-Tzu female dogs by ultrasound. Vet Med [serial on the Internet]. 2011 [cited 2019 May 10];5:1-6. Available from: http://www.journals.ufrpe.br/index.php/medicinaveterinaria/article/view/643/522

Cecchetto M, Milani C, Vencato J, Sontas H, Mollo A, Contiero B, Romagnoli S. Clinical use of fetal measurements to determine the whelping day in German shepherd breed bitches. Anim Reprod Sci. 2017;184:110-9. http://dx.doi.org/10.1016/j.anireprosci.2017.07.005. PMid:28732669.

Concannon PW, Lein DH. Hormonal and clinical correlates of ovarian cycles, ovulation, pseudopregnancy, and pregnancy in dogs. In: Kirk R, editor. Current veterinary therapy small animal practice. 10th ed. Philadelphia: Saunders; 1989. p. 1269-82.

Concannon PW, Whaley S, Lein D, Wissler R. Canine gestation length: variation related to time of mating and fertile life of sperm. Am J Vet Res. 1983;44(10):1819-21. PMid:6685444.

Federation Cynologique Internationale - FCl. FCl-Standard N²18: Chihuahua [Internet]. Belgique: FCl; 2010 [cited 2019 May 10]. Available from: http://www.fci.be/Nomenclature/Standards/218g09-en.pdf

Gaudet AD. Retrospective study of 128 cases of canine dystocia. J Am Anim Hosp Assoc. 1985;21:813-8.

Giannico AT, Gil EMU, Garcia DAA, Froes TR. The use of Doppler evaluation of the canine umbilical artery in prediction of delivery time and fetal distress. Anim Reprod Sci. 2015;154:105-12. http://dx.doi.org/10.1016/j.anireprosci.2014.12.018. PMid:25596637.

Gil EMU, Garcia DAA, Giannico AT, Froes TR. Canine fetal heart rate: do accelerations or decelerations predict the parturition day in bitches? Theriogenology. 2014;82(7):933-41. http://dx.doi.org/10.1016/j.theriogenology.2014.04.025. PMid:24888684.

Groppetti D, Vegetti F, Bronzo V, Pecile A. Breed-specific fetal biometry and factors affecting the prediction of whelping date in the German shepherd dog. Anim Reprod Sci. 2015;152:117-22. http://dx.doi.org/10.1016/j.anireprosci.2014.11.018. PMid:25510562.

Hase M, Hori T, Kawakami E, Tsutsui T. Plasma LH and progesterone levels before and after ovulation and observation of ovarian follicles by ultrasonographic diagnosis system in dogs. J Vet Med Sci. 2000;62(3):243-8. http://dx.doi.org/10.1292/jvms.62.243. PMid:10770594. 
Jabin VCP, Finardi JC, Mendes FCC, Weiss RR, Kozicki LE, Moraes R. Use of ultrasonography exams to determinate the parturition day by Yorkshire canine breed. Arch Vet Sci. 2007;12:63-70. http://dx.doi.org/10.5380/avs.v12i1.9231.

Luvoni GC, Grioni A. Determination of gestational age in medium and small size bitches using ultrasonographic fetal measurements. J Small Anim Pract. 2000;41(7):292-4. http://dx.doi.org/10.1111/j.1748-5827.2000.tb03204.x. PMid:10976623.

Melo KCM, Souza DMB, Teixeira MLB, Amorim MJAAL, Wischral A. Ultrasonographic foetal measurement to estimate the parturition time in American Cocker Spaniel and Chow-chow bitches. Vet Sci Trop [serial on the Internet]. 2006 [cited 2019 May 10];9(1):23-30. Available from: https://www.bvsvet.org.br/vetindex/periodicos/ciencia-veterinaria-nos-tropicos/9-(2006)-1/fetometria-ultrasonografica-na-previsao-da-data-do-parto-em-cadelas-d/

Nyland TJ, Mattoon JS. Small animal diagnostic ultrasound. Philadelphia: WB Saunders; 2002.

O'Neill DG, O'Sullivan AM, Manson EA, Church DB, Boag AK, McGreevy PD, Brodbelt DC. Canine dystocia in 50 UK first-opinion emergency-care veterinary practices: prevalence and risk factors. Vet Rec. 2017;181(4):88-6. http://dx.doi.org/10.1136/vr.104108. PMid:28526775.

Papp Z, Fekete T. The evolving role of ultrasound in obstetrics/gynecology practice. Int J Gynaecol Obstet. 2003;82(3):339-46. http://dx.doi.org/10.1016/S0020-7292(03)00224-8. PMid:14499980.

Pieri NCG, Souza AFD, Casals JB, Roballo KCS, Ambrósio CE, Martins DDS. Comparative development of embryonic age by organogenesis in domestic dogs and cats. Reprod Domest Anim. 2015;50(4):62531. http://dx.doi.org/10.1111/rda.12539. PMid:25990819.

Silva MRC, Sterman FDA, Almeida AH. Ultrasonographic mensuration of head dimentions in Boxers normal gestations and its correlation with the gestational age. Braz J Vet Res Anim Sci. 2007;44(Suppl.):25-9. http://dx.doi.org/10.11606/issn.1678-4456.bjvras.2007.26586.

Socha P, Janowski T. Comparison of three different fetometric formulas of ICC and BP for calculating the parturition date in a population of German Shepherd. Theriogenology. 2017;95:48-53. http://dx.doi.org/10.1016/j.theriogenology.2017.02.026. PMid:28460679.

Socha P, Janowski T. Specific fetometric formulas of ICC and BP for calculating the parturition date in the miniature breeds of canine. Reprod Domest Anim. 2018;53(2):545-9. http://dx.doi.org/10.1111/rda.13143. PMid:29399892.

Son $\mathrm{CH}$, Jeong KA, Kim JH, Park IC, Kim SH, Lee CS. Establishment of the prediction table of parturition day with ultrasonography in small pet dogs. J Vet Med Sci. 2001;63(7):715-21. http://dx.doi.org/10.1292/jvms.63.715. PMid:11503898.

Teixeira MJ, Souza DMB, Melo KCM, Wischral Á. Estimation of parturition date through ultrasonographic foetal biometry in Rottweiler bitches. Braz Anim Sci [serial on the Internet]. 2009 [cited 2019 May 10];10:853-61. Available from: https://revistas.ufg.br/vet/article/view/1828

Traas AM. Surgical management of canine and feline dystocia. Theriogenology. 2008;70(3):337-42. http://dx.doi.org/10.1016/j.theriogenology.2008.04.014. PMid:18495232.

\section{Author contributions}

CAV: Data curation, Formal analysis, Methodology, Writing - original draft \& review; RFB: Supervision, Writing - review \& editing; CEAB: Supervision; MPF: Conceptualization, Data curation, Methodology; ABN: Methodology; EAR: Methodology; IMBC: Methodology; MAAS: Methodology; ROB: Methodology; MVGL: Conceptualization, Supervision. 